\title{
Effects of Triploidization of Loquat [Eriobotrya japonica (Thunb.) Lindl.] on Flavonoids and Phenolics and Antioxidant Activities in Leaves and Flower Buds
}

\author{
Mingxiu Liu \\ College of Animal Science and Technology, Southwest University, Chongqing \\ 400716, People's Republic of China; and College of Horticulture and \\ Landscape Architecture, Southwest University, Chongqing 400716, People's \\ Republic of China \\ Peng Wang and Xu Wei \\ College of Horticulture and Landscape Architecture, Southwest University, \\ Chongqing 400716, People's Republic of China

\begin{abstract}
Qing Liu
Commonwealth Scientific and Industrial Research Organization Agriculture and Food (CSIRO), Black Mountain, ACT 2601, Australia
\end{abstract} \\ Xiaolin Li, Guolu Liang, and Qigao Guo ${ }^{1}$ \\ College of Horticulture and Landscape Architecture, Southwest University, \\ Chongqing 400716, People's Republic of China
}

Additional index words. diploid, triploid, antioxidant compounds, DPPH assay, FRAP assay

\begin{abstract}
Triploid loquat $(2 n=3 x=51)$ has stronger growth vigor and larger leaves, flowers, and fruit compared with its diploid parental plant $(2 n=2 x=34)$, but the effects of triploidization on the contents of flavonoids and phenolics in leaves and flowers, which are the most important antioxidant compounds for pharmacological applications, have not been reported. In this report, 58 triploid loquat genotypes and seven corresponding diploid parental cultivars were used to evaluate the effects of triploidization on the contents of total flavonoids and phenolics and the antioxidant activities of leaves and flower buds. The results showed that the contents of total flavonoids and phenolics and their corresponding antioxidant activities were higher in most of the triploid loquat genotypes than their diploid parents. The antioxidant activities of leaves and flower buds were significantly correlated with the total flavonoids and phenolics contents in both diploid loquat and triploid loquat. It could be inferred that triploidization could increase the contents of flavonoids and phenolics in leaves and flower buds of loquat. Notably, the contents of total flavonoids and phenolics of leaves in triploid genotype ' $\mathrm{H} 3 / 24$ ' were the highest, reaching $212.00 \mathrm{mg}$ rutin equivalent (RE)/g DW and $93.06 \mathrm{mg}$ gallic acid equivalents (GAE)/g DW, respectively, which were significantly higher than those previously reported. Such a valuable trait may be stacked with other triploid traits that are already established, such as larger vegetative organs and better tolerance to various stresses, as a feasible strategy for breeding loquat cultivars with high pharmaceutical potency.
\end{abstract}

The loquat [Eriobotrya japonica (Thunb.) Lindl.] is an evergreen subtropical fruit tree that belongs to the Rosaceae family. As a

Received for publication 6 Mar. 2019. Accepted for publication 14 May 2019.

This research was supported by the National Natural Science Foundation of China (31701876), the Fundamental Research Funds for the Central Universities (XDJK2019AA001), Key Project of Chongqing Science \& Technology Commission (cstc2018jscx-mszdX0054), and Project of Chongqing Science \& Technology Commission (cstc2017jcyjAX0433).

${ }^{1}$ Corresponding author. E-mail: qgguo@126.com. native tree, it has been cultivated for thousands of years in China, mainly for its delicious fruits (Lin et al., 2007). The dry leaves and flowers of loquat have been widely used as ingredients in traditional Chinese medicine for the treatment of chronic bronchitis, coughs, and phlegm because of their antiinflammatory, antiviral, and even antitumor effects (Chinese Pharmacopeia Commission, 2015; Huang et al., 2006; Liu et al., 2016). The major pharmacological effects of bioactive compounds in loquat are largely attributable to the presence of various triterpenes, sesquiterpenes, tannins, megastigmane and glycosides, and their numerous antioxidant compounds, such as flavonoids and phenolics (Liu et al., 2016). Previous studies have demonstrated that flavonoids and phenolics are found in the leaf, flower, and fruit tissues of loquat, and that they exhibit significantly positive correlations with antioxidant activity (Esmaeili et al., 2014; Ferreres et al., 2009; Hong et al., 2008a; Xu and Chen, 2011; Zhou et al., 2011). It has also been established that the accumulation levels of flavonoids and phenolics differ significantly among different species and cultivars of loquat (Hong et al., 2008a; Xu and Chen, 2011; Zhou et al., 2011); therefore, it is feasible to improve the contents of these secondary metabolites using breeding methods.

Polyploidy through duplication of the entire genome has long been recognized as a prominent genetic force that drives species evolution and diversification. Research has indicated that $15 \%$ of angiosperm and $31 \%$ of fern speciation events are accompanied by increased ploidy (Wood et al., 2009). Interestingly, polyploidization not only changes the morphology and physiology of plant but also increases the content of secondary metabolites (Kaensaksiri et al., 2011; Sun et al., 2011; Van Laere et al., 2011). Therefore, the polyploid could be used as an effective method of increasing the contents of medicinal components of medicinal plants. It has been previously revealed that there is a positive correlation between ploidy level and antioxidant activities because of the increased contents of some bioactive compounds in polyploid plants (Das et al., 2013; Kong et al., 2017; Nakasone et al., 1999; Xie et al., 2012).

Triploid plants are highly sterile because of the abnormalities in chromosome pairing and unbalanced chromosome segregation during meiosis. Therefore, the seedless triploid loquat has long been a research target of loquat breeders because of its higher rate of edible fruit compared with the diploid loquat with large seeds. During 1997 to 2006, we identified 225 triploid loquat genotypes $(2 n=3 x=51)$ from openpollinated progenies derived from different diploid loquat cultivars $(2 n=2 x=34)$ (Guo et al., 2007). Through numerous genetic approaches, including genome in situ hybridization, we have demonstrated that these triploid loquat genotypes mainly originated from two pathways, including autotriploidizaton and allotriploidization, with the unreduced female gametes playing a major role in loquat triploidization (Liang, 2006). The triploid loquat has developmental and phenological periods that are generally similar to those of the diploid loquat. However, compared with diploid loquat, the triploid loquat has relatively stronger growth vigor because of its larger canopy and organs, such as its leaves and flowers (Liang et al., 2011a, 2011b). Moreover, compared with its diploid parent, seedless fruits have higher edible rates of $86 \%$ to $90 \%$ (Liang, 2006). Such genetic materials are excellent research subjects when studying the effects of triploidization on 
antioxidant activities and related contents of flavonoids and phenolics in loquat.

In the present research, the accumulation levels of flavonoids and phenolics were measured in the leaves and flower buds of 58 triploid loquat genotypes and their seven corresponding diploid parental cultivars. The antioxidant capacities of the leaves and flower buds were also assessed using the ferric reducing antioxidant power (FRAP) assay and the $\alpha, \alpha$-diphenyl- $\beta$-picrylhydrazyl (DPPH) free radical-scavenging activity assay, which are two common antioxidant assays. Finally, correlations were examined between the antioxidant capacity and total contents of flavonoids and phenolics in leaves and flower buds of triploid loquat and diploid loquat. To our best knowledge, this is the first attempt to evaluate the effects of triploidization on biochemical accumulations in loquat as an important medicinal plant.

\section{Materials and Methods}

\section{Plant materials}

Since 1997, 58 triploid loquat genotypes $(2 n=3 x=51)$ have been identified from diploid parental loquat cultivars $(2 n=2 x=34)$ (Table 1) (Guo et al., 2007; Liang, 2006; Liang et al., 2011a). In 2002, scions of the 58 triploid loquat genotypes and their seven diploid parental loquat cultivars were grafted onto 2year-old 'Jiefangzhong' loquat seedling rootstocks at the Loquat Research Orchard of Southwest University in Chongqing, China. Since then, both triploid genotypes and their corresponding diploid parents were grown at the Loquat Germplasms Nursery in Southwest University. Samples of the trees, shoots, leaves, flowers, and fruits of the typical triploid loquat genotype ' $\mathrm{H} 3 / 24$ ' and the corresponding diploid parent 'Ruantiaobaisha' are illustrated in Fig. 1, whereas the other six diploid parental loquats and the corresponding triploid loquat genotypes are illustrated in Supplemental Figs. 1-6. Three individual trees were selected for each genotype, and the fully expanded young leaves and flower buds were harvested in Nov. 2016. Ten leaves and 100 flower buds were sampled from each tree.

\section{Sample extraction}

The collected leaf and flower bud samples were dried for $7 \mathrm{~d}$ at $37{ }^{\circ} \mathrm{C}$ until the weight remained constant; then, they were ground into powder and run through 80 mesh sieves. open-pollinated progenies derived from seven

The dried powder $(0.5 \mathrm{~g})$ from each sample was suspended in $20 \mathrm{~mL}$ of petroleum ether and sonicated for $30 \mathrm{~min}$ using an Ultrasonic Cleaner (SB-5200D; Scientz Biotechnology Co. Ltd., Ningbo, China). Following centrifugation at $5000 g_{\mathrm{n}}$ for $5 \mathrm{~min}$, petroleum ether supernatant was removed. The pellet was resuspended in $20 \mathrm{~mL} \mathrm{70 \%} \mathrm{ethanol} \mathrm{for} \mathrm{further}$ ultrasonic treatment, which was followed by centrifugation at $10,000 g_{\mathrm{n}}$ for $5 \mathrm{~min}$ before the supernatant was collected. Following a repeat of the aforementioned process, the supernatants of the two centrifugations were used to determine the contents of total phenolics and total flavonoids and antioxidant activity.

\section{Analysis of total flavonoids}

Total flavonoids were measured as previously described, with minor modifications (Jia et al., 1999). In brief, the extract $(0.5 \mathrm{~mL})$ of each sample prepared as described was added to $300 \mu \mathrm{L}$ of $5 \% \mathrm{NaNO}_{2}$ solution and allowed to stand for $6 \mathrm{~min}$ for the reaction. This was followed by adding $300 \mu \mathrm{L}$ of $10 \%$ $\mathrm{Al}\left(\mathrm{NO}_{3}\right)_{3}$ solution, which was mixed well and allowed to stand for another $6 \mathrm{~min}$. Finally, $4 \mathrm{~mL}$ of $1.0 \mathrm{M} \mathrm{NaOH}$ was added and adjusted to a total volume of $5 \mathrm{~mL}$ with deionized water before being mixed thoroughly. The mixture solution was allowed to stand for $15 \mathrm{~min}$ at room temperature before total flavonoids were measured by a mass spectrophotometer at an absorbance $510 \mathrm{~nm}$ and expressed as $\mathrm{mg} / \mathrm{g}$ dry weight (DW) of rutin equivalents (RE).

\section{Analysis of total phenolics}

The total phenolics content was determined spectrophotometrically according to the Folin-Ciocalteu method (Singleton et al., 1999). Briefly, $0.5 \mathrm{~mL}$ of the extract solution of each sample obtained as described was diluted with $4.5 \mathrm{~mL}$ of deionized water; then, $0.25 \mathrm{~mL}$ was added to $0.5 \mathrm{~mL} 1 \mathrm{~N}$ FolinCiocalteu reagent (Sigma-Aldrich, St. Louis, MO). Following vigorous shaking, $4 \mathrm{~mL}$ of $7.5 \% \mathrm{Na}_{2} \mathrm{CO}_{3}$ solution was added to the sample. Finally, the sample volume was adjusted to $5 \mathrm{~mL}$ with deionized water and allowed to stand for $30 \mathrm{~min}$ at $25^{\circ} \mathrm{C}$; intermittent shaking was performed. The absorption was measured at $760 \mathrm{~nm}$ against deionized water as a blank. The total phenolics content of the samples was expressed as $\mathrm{mg} / \mathrm{g}$ DW of gallic acid equivalents (GAE).

\section{Analysis of antioxidant activity \\ Assay for DPPH radical scavenging activity. The DPPH free radical-scavenging}

Table 1. Diploid and corresponding triploid loquat plants used in the study.

\begin{tabular}{ll}
\hline Diploid cultivar name & \multicolumn{1}{c}{ Triploid genotypes } \\
\hline Dawuxing & $\mathrm{A} 3 / 22, \mathrm{~A} 3 / 32, \mathrm{~A} 3 / 48, \mathrm{~A} 3 / 13, \mathrm{~A} 3 / 30, \mathrm{~A} 3 / 68, \mathrm{~A} 3 / 5, \mathrm{~A} 3 / 71, \mathrm{~A} 3 / 84, \mathrm{~A} 3 / 76$ \\
Longquan no.1 & $\mathrm{B} 3 / 16, \mathrm{~B} 3 / 50, \mathrm{~B} 3 / 8, \mathrm{~B} 3 / 52, \mathrm{~B} 3 / 77, \mathrm{~B} 3 / 78, \mathrm{~B} 3 / 72, \mathrm{~B} 3 / 55, \mathrm{~B} 3 / 40, \mathrm{~B} 3 / 70, \mathrm{~B} 3 / 37$, \\
& $\mathrm{B} 3 / 45, \mathrm{~B} 3 / 56, \mathrm{~B} 3 / 47, \mathrm{~B} 3 / 39, \mathrm{~B} 3 / 73, \mathrm{~B} 3 / 57, \mathrm{~B} 3 / 53, \mathrm{~B} 4 / 41, \mathrm{~B} 3 / 65, \mathrm{~B} 4 / 14$, \\
& $\mathrm{B} 4 / 3 / 31$ \\
Jinfeng & $\mathrm{D} 27, \mathrm{D} 29, \mathrm{D} 36, \mathrm{D} 4 / 25$ \\
77-1 & $\mathrm{K} 3 / 59, \mathrm{~K} 2 / 380, \mathrm{~K} 3 / 63$, offspring of K3/75, K3/64, K3/75, K3/46, K3/74, K3/51 \\
Xiangzhong & $\mathrm{N} 18, \mathrm{~N} 28, \mathrm{~N} 17, \mathrm{~N} 22, \mathrm{~N} 13, \mathrm{~N} 14, \mathrm{~N} 26$ \\
Changbai no.1 & $\mathrm{Q} 11, \mathrm{Q} 21, \mathrm{Q} 16, \mathrm{Q} 27, \mathrm{Q} 24$ \\
Ruantiaobaisha & $\mathrm{H} 3 / 24$ \\
\hline
\end{tabular}

activity was determined according to the method of Brand-Williams et al. (1995). In brief, a $100-\mu \mathrm{L}$ aliquot of the previously diluted extract was allowed to react with $2.9 \mathrm{~mL}$ of DPPH $(0.5 \mathrm{~mm})$ for $30 \mathrm{~min}$ in the dark. Then, the absorbance was detected at a wavelength of $515 \mathrm{~nm}$. Results were expressed as $\mu \mathrm{M} / \mathrm{g}$ DW of trolox equivalents (TE).

Assay for ferric reducing antioxidant power. The FRAP assay was performed according to the method of Benzie and Strain (1996). In brief, each diluted sample $(0.1 \mathrm{~mL})$ was added to $2.9 \mathrm{~mL}$ of the freshly prepared FRAP reagent solution $(0.1 \mathrm{M}$ acetate buffer, $\mathrm{pH}$ 3.6, $10 \mathrm{~mm}$ TPTZ, and $20 \mathrm{~mm}$ ferric chloride, 10:01:01, v/v/v) and incubated at $37^{\circ} \mathrm{C}$ for $10 \mathrm{~min}$. Absorbance at $593 \mathrm{~nm}$ was measured against the reagent blank $(2.9 \mathrm{~mL}$ of the FRAP reagent plus $0.1 \mathrm{~mL}$ of deionized water) and expressed as $\mu \mathrm{M} / \mathrm{g}$ DW of TE.

\section{Statistical analysis}

All data were collected as the means $\pm \mathrm{SD}$ of three biological replicates. Statistical analyses were performed using statistical package JMP 10.0.0 (SAS Institute, Cary, NC). Significant differences among all genotypes tested were calculated using a one-way analysis of variance test. Differences were considered statistically significant at $P<0.05$.

\section{Results}

\section{Variations in the contents of total flavonoids and phenolics in leaves and flower buds of seven diploid parental loquat cultivars}

The total flavonoids and phenolics contents were determined by the spectrophotometric method. Results showed that the variations of the total flavonoids and phenolics contents in leaves and flower buds were significantly different among the seven parental diploid loquat varieties (Fig. 2).

Among the diploid parental loquat cultivars, the highest total flavonoid content in leaves was $162.20 \mathrm{mg} \mathrm{RE} / \mathrm{g} \mathrm{DW}$ in 'Xiangzhong', which was $63 \%$ higher than the lowest level (99.75 $\mathrm{mg} \mathrm{RE} / \mathrm{g} \mathrm{DW}$ ) in 'Changbai no. 1'. The highest accumulation of flavonoids in flower buds was found in 'Ruantiaobaisha' and was $228.74 \mathrm{mg} \mathrm{RE} / \mathrm{g}$ DW); that value was two-times higher than the lowest level found in 'Longquan no. 1' (110.91 mg RE/g DW). The accumulation patterns of total phenolics and total flavonoids were similar in leaves of the seven parental diploid loquat cultivars as well as in the flower buds. For example, Xiangzhong also possessed the highest total phenolic content in leaves, at $77.61 \mathrm{mg} \mathrm{GAE} / \mathrm{g} \mathrm{DW}$, and that value was $40 \%$ higher than the lowest level (55.51 $\mathrm{mg} \mathrm{GAE} / \mathrm{g}$ DW) found in the same tissue in 'Longquan no. 1'. In flower buds, the highest phenolics content was found in 'Changbai no. 1' and was $84.37 \mathrm{mg} \mathrm{GAE} / \mathrm{g} \mathrm{DW}$; that value was $28 \%$ higher than the lowest level (65.70 mg GAE/g DW) in 'Longquan no. 1'. Generally, the total accumulation levels of flavonoids and phenolics in flower buds were 

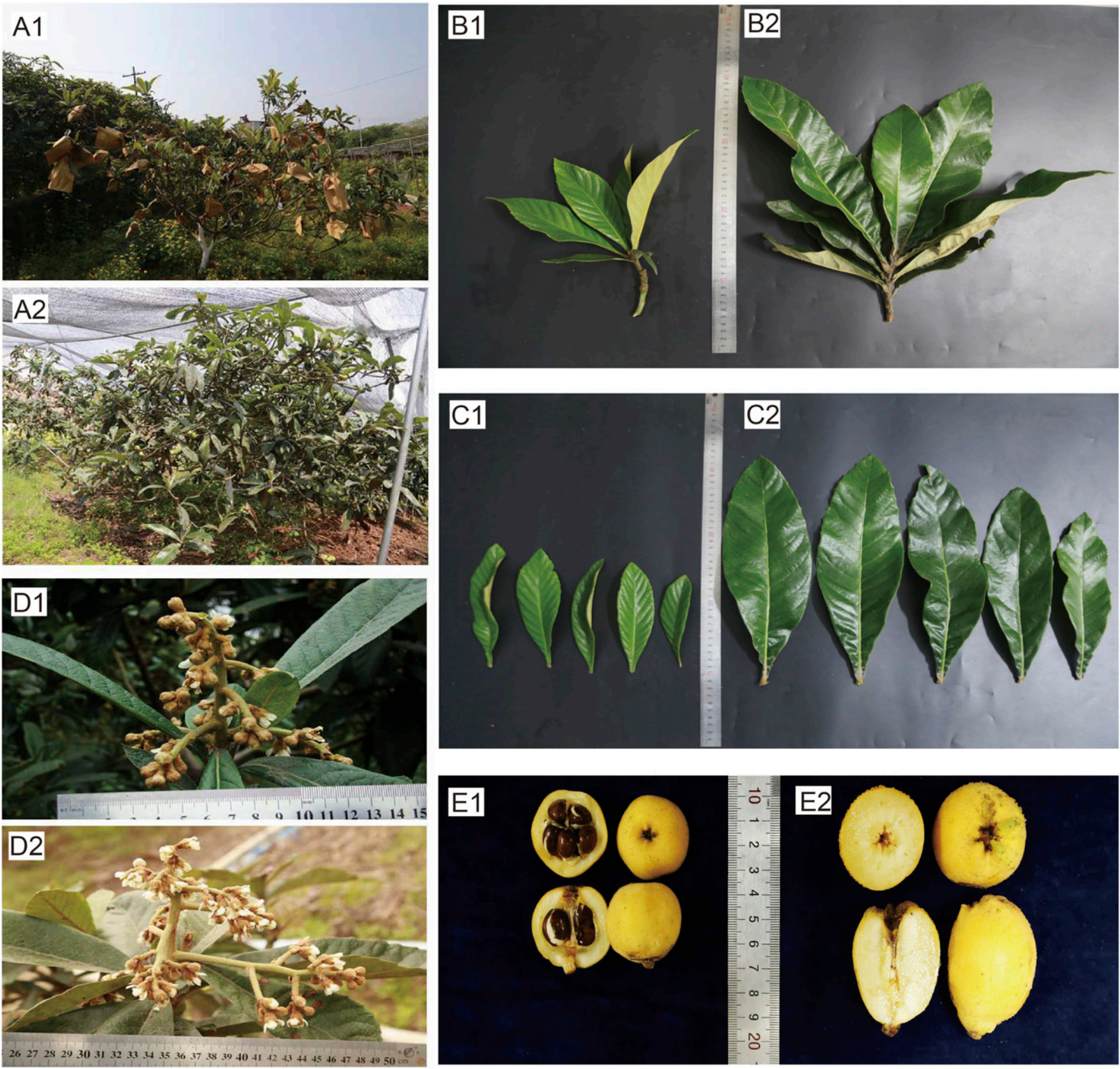

Fig. 1. The trees, shoots, leaves, flowers, and fruits of the triploid loquat genotype 'H3/24' and corresponding diploid loquat 'Ruantiaobaisha'. (A1) Tree of 'Ruantiaobaisha'. (A2) Tree of 'H3/24'. (B1) Shoots of 'Ruantiaobaisha'. (B2) Shoots of 'H3/24'. (C1) Leaves of 'Ruantiaobaisha'. (C2) Leaves of 'H3/24'. (D1) Flowers of 'Ruantiaobaisha'. (D2) Flowers of 'H3/24'. (E1) Fruits of 'Ruantiaobaisha'. (E2) Fruits of 'H3/24'

higher than those in leaves in the diploid parental loquat, except for 'Xiangzhong' (Fig. 2).

\section{Comparison of the total flavonoids and phenolics contents in leaves and flower buds of triploid loquat genotypes}

The contents of total flavonoids and phenolics in triploid genotypes showed a generally significant alteration compared with their diploid parents (Table 2). Approximately $43 \%$ and $89 \%$ triploid genotypes derived, respectively, from 'Xiangzhong' and ' $77-1$ ' exhibited lower accumulations of total flavonoids in leaves compared with their respective diploid parents. Approximately $90 \%$ of triploid genotypes of 'Dawuxing' did not show significant differences compared with its diploid parents $(P<0.01)$. In contrast, the total flavonoid contents in the leaves of the diploid parents of 'Jingfeng' and 'Longquan no. 1' were low and were significantly increased in corresponding triploid genotypes $(P<0.05)$. The contents of the total flavonoids in the leaves of all the triploid genotypes of 'Longquan no. 1' were also higher than those of their diploid parents, with $\approx 41 \%$ of genotypes showing statistical significance $(P<0.05)$. 'Changbai no. 1' and 'Ruantiaobaisha' are white-flesh loquat; $80 \%$ triploid genotypes of 'Changbai no. 1' showed a significant enhancement of the total flavonoids content in leaves $(P<0.01)$, with the maximum increase reaching $156.84 \mathrm{mg}$
RE/g DW for 'Q24' (57\% higher than the diploid parent). ' $\mathrm{H} 3 / 24$ ', the only triploid genotype derived from 'Ruantiaobaisha', exhibited the highest contents of total flavonoids in leaves (212.00 mg RE/g DW) among all triploid genotypes (Table 2). In flower buds, the contents of the total flavonoids were higher than those in the leaves of all triploid loquat genotypes, except for 'H324'. The total flavonoid contents in the flower buds of most triploid genotypes were significantly enhanced compared with their diploid parents. Approximately $86 \%, 33 \%, 80 \%, 75 \%$, $95 \%$, and $80 \%$ triploid genotypes derived, respectively, from 'Xiangzhong', '77-1', 'Dawuxing', 'Jingfeng', 'Longquan no. 1', and 'Changbai no. 1' showed higher accumulations 

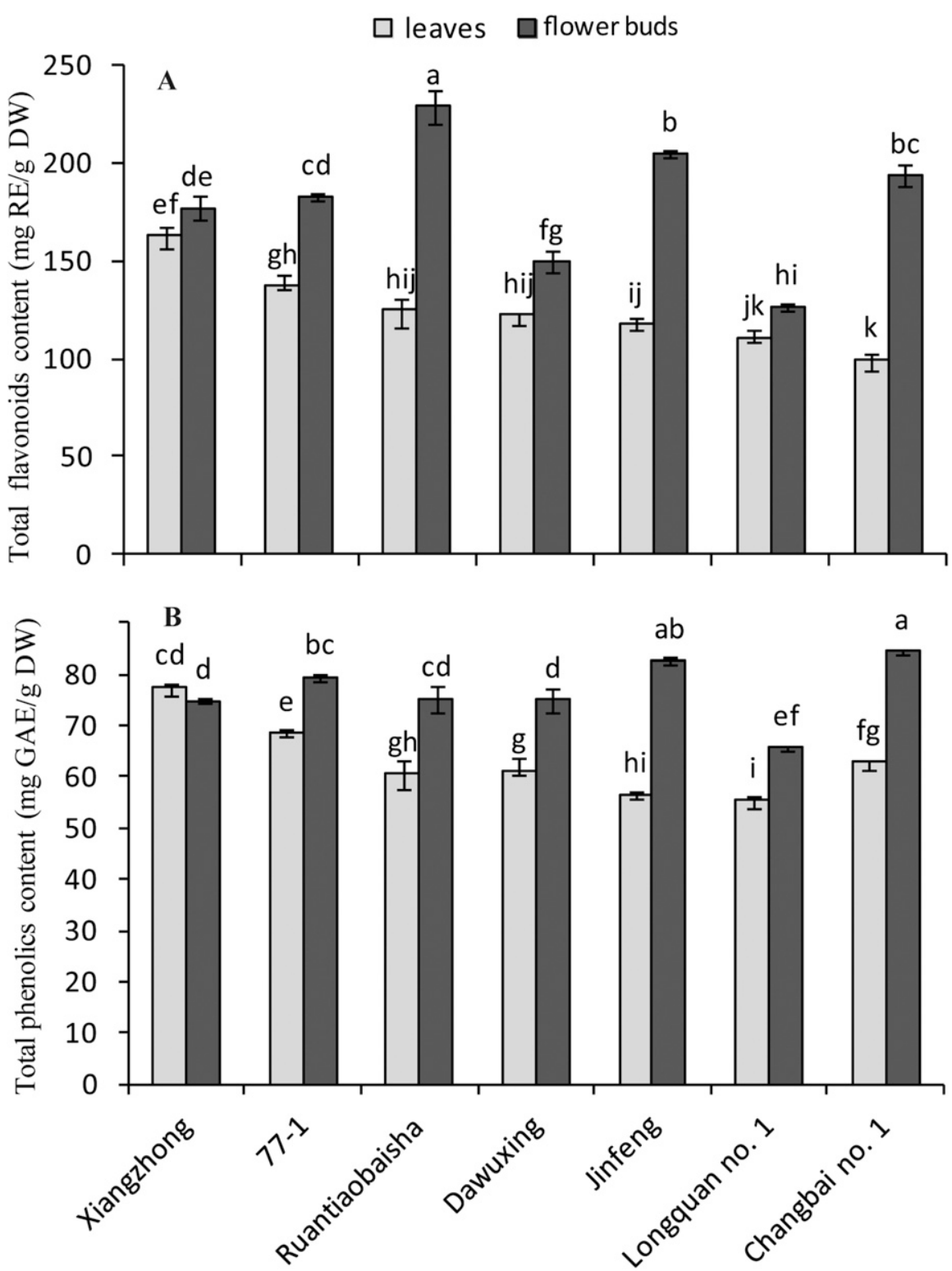

Fig. 2. Total flavonoids and phenolics contents in extracts of leaves and flower buds of the seven diploid parental loquat cultivars. (A) Total flavonoids content. (B) Total phenolics content. Significant differences between means were determined using Tukey's honestly significant difference test $(\mathrm{n}=3)$. Different letters above the bars indicate that the means are significantly different $(P<0.05)$. Values are presented as mean $( \pm \mathrm{SD})$.

of total flavonoids in flower buds compared with their respective diploid parents $(P<$ 0.05 ) (Table 2). The maximum increase was recorded as $246.82 \mathrm{mg} \mathrm{RE} / \mathrm{g} \mathrm{DW}$ for ' $\mathrm{A} 3 / 76$ ' (65\% higher than the diploid parent). In contrast, the total flavonoids content of triploid genotypes in flower buds of ' $\mathrm{H} 3 / 24$ ' decreased significantly compared with its diploid parent $(P<0.01)$ (Table 2$)$.

The total phenolics content of leaves in the diploid parent loquat ranged from 55.51 to $77.61 \mathrm{mg} \mathrm{GAE} / \mathrm{g} \mathrm{DW}$, and those in triploid loquat genotypes ranged from 47.20 to $93.06 \mathrm{mg} \mathrm{GAE} / \mathrm{g} \mathrm{DW}$. The contents of the total phenolics in flower buds were generally higher than those in leaves in most triploid loquat genotypes. The total phenolics contents of flower buds were from 65.70 to
(449.46 $\mu \mathrm{M}$ TE/g DW) in leaves; this value was almost $50 \%$ higher than the lowest DPPH value found in 'Ruantiaobaisha' $(300.35 \mu \mathrm{M}$ $\mathrm{TE} / \mathrm{g} \mathrm{DW})$. The antioxidant activities of the extracts derived from flower buds were generally higher than those from leaves. In flower buds, 'Ruantiaobaisha' showed the highest DPPH value $(677.93 \mu \mathrm{M} \quad \mathrm{TE} / \mathrm{g}$ $\mathrm{DW})$; that value was $56 \%$ higher than the lowest DPPH value in 'Longquan no. 1' (434.64 $\mu \mathrm{M}$ TE/g DW). It is interesting that the antioxidant activity values measured by the FRAP assay were consistently higher than the corresponding DPPH values.

Comparisons of antioxidant activities of the extracts derived from the leaves and flower buds of diploids and corresponding triploid loquat genotypes

$D P P H$. The DPPH values of the leaf extracts of triploid loquat genotypes ranged from 228.85 to $589.86 \mu \mathrm{M}$ TE/g DW, and those from the seven diploid parental genotypes varied from 300.35 to $449.46 \mu \mathrm{M} \mathrm{TE} / \mathrm{g}$ DW (Table 3). However, '77-1' had the highest DPPH value (449.46 $\mu \mathrm{M}$ TE/g DW) among the seven diploid parental loquat varieties, followed by 'Xiangzhong'. The corresponding triploid genotypes of these two diploids showed a $78 \%$ reduction and a $43 \%$ reduction, respectively, in antioxidant activities compared with their respective diploid parents $(P<0.01)$. In contrast, $90 \%$ triploid plants derived from 'Dawuxing' showed either no significant difference or a significant reduction in the DPPH value compared with its diploid parent $(P<0.05)$. In contrast, the triploids derived from the diploids with low DPPH values, such as 'Jingfeng', 'Changbai no. 1', and 'Longquan no. 1 ', showed significantly increased DPPH values, with $100 \%, 80 \%$, and $59 \%$ increments, respectively, in triploid plants compared with their corresponding diploid parents $(P<0.05)$. 'H3/24', the only triploid genotype derived from 'Ruantiaobaisha', also showed a significant increase in the DPPH value compared with its diploid parent $(P<0.01)$.

In both triploids and their diploid parents, the DPPH values of loquat in flower buds were higher than those in leaves. The DPPH values of the flower buds varied between 434.64 and $677.93 \mu \mathrm{M}$ TE/g DW in diploids, and they varied between 415.56 and $777.77 \mu \mathrm{M}$ TE/g DW in triploids (Table 3). The DPPH values of the flower buds in most triploid genotypes showed a significant increase relative to their diploid parents, except for 'Changbai no. 1' and 'Ruantiaobaisha'. The maximum DPPH value $(777.77 \mu \mathrm{M} \mathrm{TE} / \mathrm{g}$ DW) was observed in 'B3/70', which increased by $79 \%$ compared with its diploid parent.

FRAP. The FRAP assay is usually used to measure the capacity of the ferric complex to reduce the ferrous form in plant cells (Benzie and Strain, 1996). The FRAP values of the leaf extract varied between 371.33 and $599.84 \mu \mathrm{M}$ TE/g DW in diploids, and they varied between 322.83 and $731.36 \mu \mathrm{M}$ TE/g 
Table 2. Contents of total flavonoids and phenolics in leaves and flower buds of diploid and corresponding triploid loquat plants.

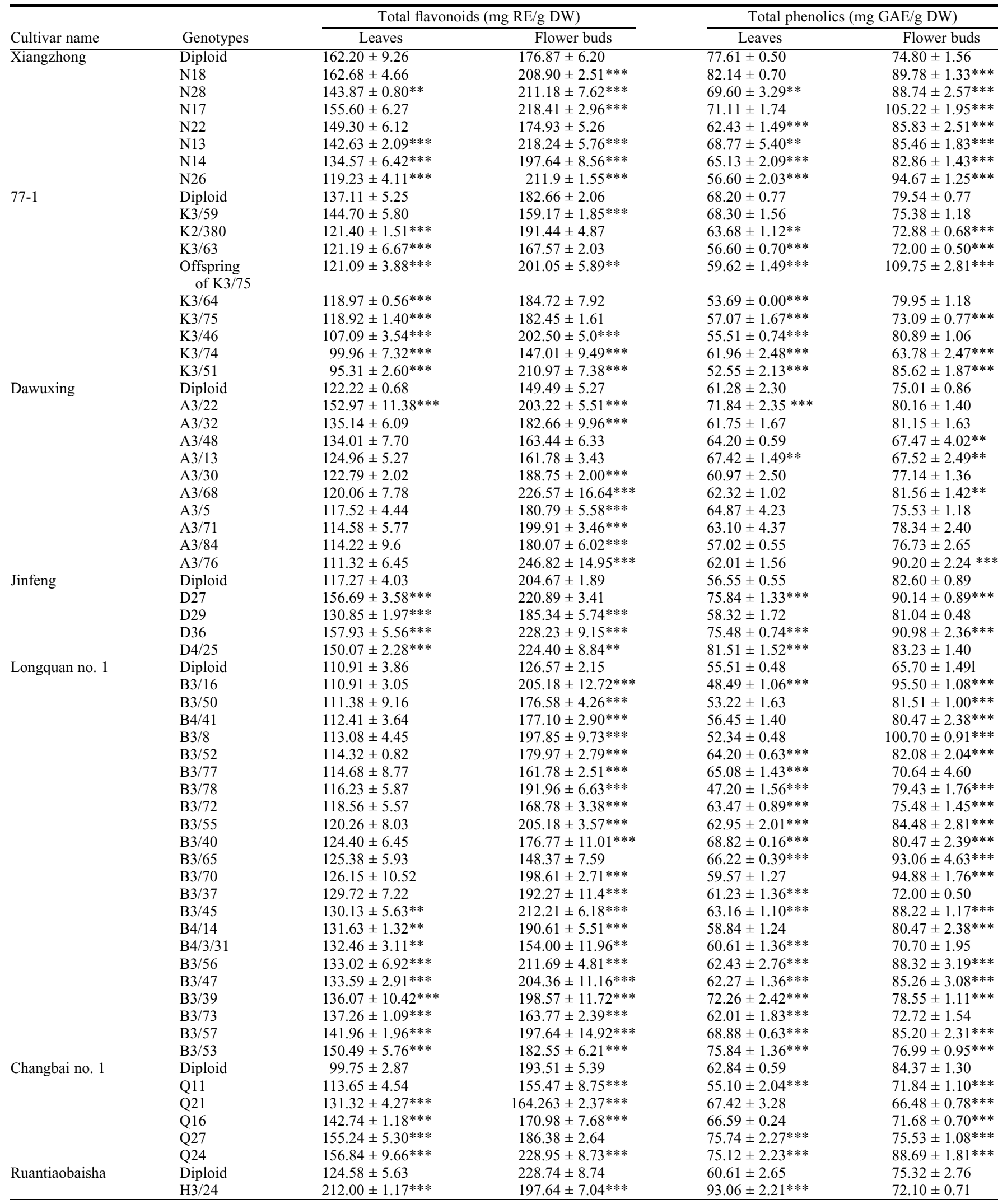

The values represent the mean $\pm \mathrm{SD}(\mathrm{n}=3)$.

Based on Tukey's honestly significant difference test after one-way analysis of variance, statistically significant events compared with corresponding diploids are indicated as $* * *, P<0.01$ and $* *, P<0.05$.

DW in triploids (Table 3). Similar to the DPPH results, 'Xiangzhong', '77-1', and 'Dawuxing' showed relatively higher FRAP values compared to other diploid varieties
$(P<0.05)$. Almost all of their corresponding triploid genotypes showed reductions in FRAP values compared with their respective diploids $(P<0.05)$. This was in contrast to those with relatively lower FRAP values, such as 'Jingfeng', 'Longquan no. 1', and 'Changbai no. 1'. Most of these triploid genotypes showed significant increases in 

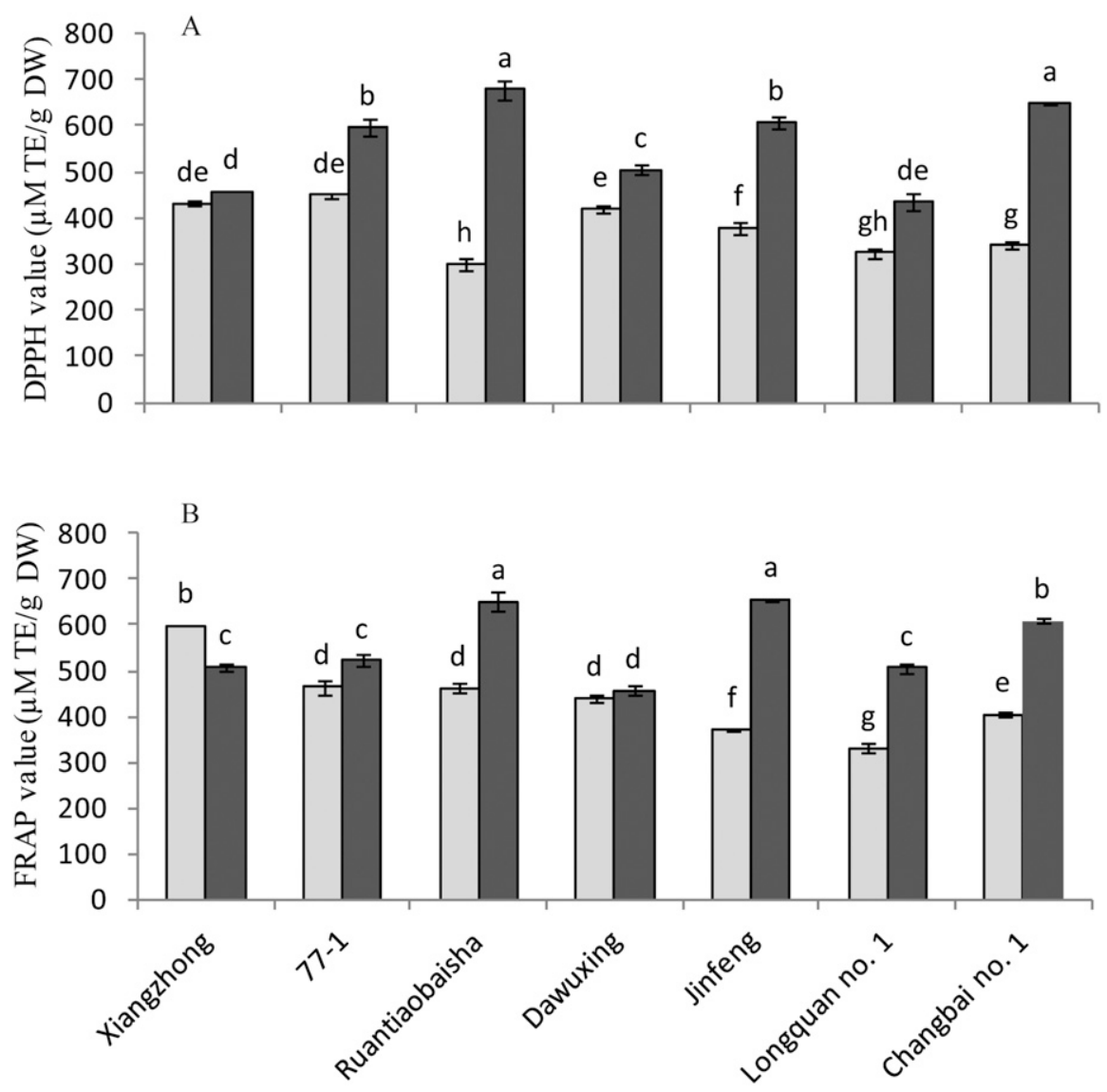

Fig. 3. Antioxidant activities in extracts of leaves and flower buds of the seven diploid parental loquat cultivars. (A) DPPH value. (B) FRAP value. Significant differences between means were determined using Tukey's honestly significant difference test $(\mathrm{n}=3)$. Different letters above the bars indicate that the means are significantly different $(P<0.05)$. Values are presented as mean $( \pm \mathrm{SD})$.

the FRAP values of leaf extracts compared with their respective diploid parents $(P<$ $0.01)$. 'H3/24' showed the highest FRAP value of leaf extracts $(731.33 \mu \mathrm{M}$ TE/g DW).

The FRAP value of flower buds was generally higher than those of leaves, and it varied between 456.81 and $656.83 \mu \mathrm{M}$ TE/g DW in diploids and between 446.40 and $888.64 \mu \mathrm{M}$ TE/g DW in triploids. The FRAP values of triploid genotypes in flower buds were higher than those in most of their corresponding diploid parents. The maximum FRAP value was recorded as $888.64 \mu \mathrm{M}$ TE/g DW for 'A3/76', which increased by $95 \%$ compared with its diploid parent.

\section{Correlation analysis between total flavonoids or phenolics contents and antioxidant activities}

The linear correlation coefficient analysis indicated that there was a significant positive correlation between the total flavonoids, total phenolics, and antioxidant activities of leaves and flower buds in both diploid loquat and triploid loquat (Table 4). Therefore, the total phenolics and flavonoids presented in the leaves and flower buds of loquat could be the major antioxidants that participated in scavenging free radicals when incorporated in Chinese medicine. However, the correlation coefficients between different tissues, ploidy levels, and antioxidant assays showed considerable variations. For example, the total flavonoids and phenolics in the leaf of diploid loquat were significantly correlated with DPPH ( $P<0.05, r=0.60, r=0.60$, respectively $)$, and an even stronger positive correlation was obtained with FRAP $(P<0.01, r=0.85$, $r=0.91$, respectively). In diploid loquats, the total contents of flavonoids in flower buds were also significantly correlated with DPPH $(P<0.01, r=0.86)$ and FRAP $(P<$ $0.01, r=0.83)$, but the total contents of phenolics showed relatively lower levels of correlation with either DPPH or FRAP. In the triploid loquats, the total flavonoids and phenolics in leaves presented significant positive correlations with DPPH $(P<0.01$, $r=0.73, r=0.71$, respectively), with correlation coefficients significantly higher than those in diploid loquats. Although the correlation coefficients of FRAP $(P<0.01$, $r=0.81, r=0.86$, respectively) were similar between triploid and diploid plants, the total flavonoids and phenolics in flower buds showed relatively lower levels of correlation with either DPPH or FRAP compared with leaves.
Numerous studies have demonstrated that the increase in ploidy levels could lead to enhanced production of metabolites and significant increases in the contents of secondary metabolites in polyploids, especially the induced autopolyploid lines (Kaensaksiri et al., 2011; Lin et al., 2011; Sun et al., 2011; Van Laere et al., 2011). For example, the contents of phenolic acids and flavonoids were significantly increased in Lonicera japonica following autopolyploidization compared with its diploid parent (Kong et al., 2017). Similarly, in the fruit pulp of Hylocereus (Cactaceae), several flavonoids showed significant increases following autopolyploidization (Cohen et al., 2013). The contents of flavonoids in the leaves of tetraploid Dracocephalum kotschyi were significantly increased relative to corresponding diploid plants (Zahedi et al., 2014). However, polyploidization as a strategy to enhance metabolite production could not be generalized. In contrast to the aforementioned examples, tetraploid potato genotypes displayed metabolites that were similar to or lower than those of their diploid parents (Caruso et al., 2013).

A triploid plant is uncommon in nature because of its high level of sterility, but some research showed that the triploid plants have an important bridge role in the process of tetraploid formation (Kovalsky et al., 2017; Ramsey and Schemske, 1998). The mechanism, which involves reduced and an unreduced gamete fusion, is the generally accepted theory for triploid formation among diploid populations (Bretagnolle and Thompson, 1995). In addition, polyspermy has been proposed for some plants (Toda and Okamoto, 2016). However, the spontaneous frequency of unreduced gametes is low in loquat, and the unreduced pollen frequency is only $2.64 \%$, even though the inflorescence of diploid loquat was induced by "hot shock" (Wang et al., 2003). Therefore, the low frequency of unreduced gametes is still a substantial hurdle in triploid breeding. Because tetraploid usually produces reduced gametes $(n=2 x)$, hybridization between a tetraploid and a diploid has become a feasible approach to generating triploid hybrid progeny (Kikuchi et al., 2014). However, the pollen viability and fruit set rate of the tetraploid are key limiting factors for altered ploidy-level hybridization. Therefore, the evaluation and utilization of foundation tetraploid parents will be particularly important to improving the frequency of triploid occurrence. It was found that the average frequency of triploidization reached $66.67 \%$ in 10 reciprocal cross-combinations with 'B431' as the main tetraploid parent (Liang et al., 2018). Therefore, directional hybridization using diploids and tetraploids is an effective strategy for generating triploid loquat. Generally, studies of metabolism in triploids have been relatively rare compared with other polyploids because of their scarcity. To the best of our knowledge, only one report has described a diverse metabolite 
Table 3. Antioxidant activities of extracts from leaves and flower buds of diploid and corresponding triploid loquat plants.

\begin{tabular}{|c|c|c|c|c|c|}
\hline \multirow[b]{2}{*}{ Cultivar name } & \multirow[b]{2}{*}{ Genotypes } & \multicolumn{2}{|c|}{ DPPH $(\mu \mathrm{M}$ TE/g DW) } & \multicolumn{2}{|c|}{ FRAP $(\mu \mathrm{M}$ TE/g DW $)$} \\
\hline & & Leaves & Flower buds & Leaves & Flower buds \\
\hline \multirow{4}{*}{ Xiangzhong } & N18 & $579.83 \pm 9.06^{* * *}$ & $644.79 \pm 20.11 * * *$ & $633.00 \pm 0.95$ & $614.64 \pm 19.56^{* * *}$ \\
\hline & $\mathrm{N} 22$ & $517.92 \pm 14.70^{* * *}$ & $697.98 \pm 9.99 * * *$ & $493.53 \pm 13.05^{* * *}$ & $715.47 \pm 3.77^{* * *}$ \\
\hline & N13 & $354.42 \pm 11.87 * * *$ & $764.26 \pm 6.92 * * *$ & $488.32 \pm 8.40^{* * *}$ & $750.82 \pm 14.26^{* * *}$ \\
\hline & N14 & $380.14 \pm 15.33 * * *$ & $588.11 \pm 10.97 * * *$ & $475.99 \pm 14.92 * * *$ & $712.46 \pm 9.53^{* * *}$ \\
\hline \multirow{6}{*}{$77-1$} & $\mathrm{~K} 3 / 59$ & $432.46 \pm 9.46$ & $548.10 \pm 9.82 * * *$ & $481.75 \pm 10.41$ & $683.96 \pm 8.63^{* * *}$ \\
\hline & $\mathrm{K} 2 / 380$ & $381.45 \pm 1.51^{* * *}$ & $591.27 \pm 5.29$ & $430.24 \pm 19.75$ & $477.36 \pm 9.02$ \\
\hline & $\mathrm{K} 3 / 63$ & $306.46 \pm 7.96^{* * *}$ & $503.20 \pm 5.70^{* * *}$ & $432.43 \pm 28.62$ & $446.40 \pm 14.15^{* * *}$ \\
\hline & $\begin{array}{l}\text { Offspring } \\
\text { of } \mathrm{K} 3 / 75\end{array}$ & $491.32 \pm 14.11^{* *}$ & $701.14 \pm 2.00 * * *$ & $457.91 \pm 21.82$ & $846.72 \pm 16.32^{* * *}$ \\
\hline & $\mathrm{K} 3 / 64$ & $298.61 \pm 15.08 * * *$ & $627.46 \pm 14.86^{* *}$ & $333.79 \pm 26.97 * * *$ & $719.58 \pm 12.84^{* * *}$ \\
\hline & $\mathrm{K} 3 / 75$ & $382.76 \pm 12.36 * * *$ & $512.79 \pm 20.78^{* * *}$ & $444.76 \pm 35.03$ & $484.49 \pm 23.20^{* * *}$ \\
\hline \multirow{9}{*}{ Dawuxing } & $\mathrm{A} 3 / 32$ & $400.63 \pm 9.06$ & $607.70 \pm 5.45^{* * *}$ & $377.90 \pm 15.30^{* * *}$ & $637.11 \pm 6.69^{* * *}$ \\
\hline & $\mathrm{A} 3 / 48$ & $402.81 \pm 4.95$ & $524.86 \pm 15.98$ & $466.95 \pm 3.88^{* *}$ & $597.92 \pm 11.39^{* * *}$ \\
\hline & $\mathrm{A} 3 / 13$ & $395.40 \pm 6.92$ & $585.46 \pm 14.41^{* * *}$ & $487.50 \pm 4.14^{* * *}$ & $536.27 \pm 20.19^{* * *}$ \\
\hline & $\mathrm{A} 3 / 30$ & $342.64 \pm 12.36^{* * *}$ & $613.80 \pm 4.00 * * *$ & $427.50 \pm 4.67$ & $672.73 \pm 17.57 * * *$ \\
\hline & $\mathrm{A} 3 / 68$ & $375.34 \pm 2.00 * *$ & $693.16 \pm 3.46 * * *$ & $408.32 \pm 7.46^{* * *}$ & $850.00 \pm 33.05^{* * *}$ \\
\hline & $\mathrm{A} 3 / 5$ & $383.19 \pm 18.97$ & $606.39 \pm 12.15^{* * *}$ & $449.42 \pm 7.37$ & $531.07 \pm 6.89^{* * *}$ \\
\hline & $\mathrm{A} 3 / 71$ & $392.35 \pm 19.06$ & $595.49 \pm 10.57 * * *$ & $420.65 \pm 2.07$ & $555.73 \pm 11.66^{* * *}$ \\
\hline & $\mathrm{A} 3 / 84$ & $402.81 \pm 4.95$ & $697.95 \pm 8.71 * * *$ & $399.82 \pm 1.64^{* * *}$ & $634.64 \pm 23.33^{* * *}$ \\
\hline & $\mathrm{A} 3 / 76$ & $300.35 \pm 10.49 * * *$ & $738.5 \pm 24.62 * * *$ & $380.64 \pm 7.09^{* * *}$ & $888.64 \pm 40.02 * * *$ \\
\hline \multirow[t]{4}{*}{ Jinfeng } & Diploid & $378.83 \pm 14.41$ & $606.86 \pm 12.43$ & $371.33 \pm 3.11$ & $656.83 \pm 2.64$ \\
\hline & D27 & $519.22 \pm 20.70^{* * *}$ & $721.96 \pm 9.28 * * *$ & $516.55 \pm 10.87^{* * *}$ & $712.73 \pm 6.17^{* * *}$ \\
\hline & D29 & $383.63 \pm 2.27 * *$ & $619.07 \pm 11.77$ & $442.29 \pm 2.51 * * *$ & $639.85 \pm 1.64$ \\
\hline & D36 & $438.56 \pm 4.53 * * *$ & $752.48 \pm 2.27 * * *$ & $589.70 \pm 5.39^{* * *}$ & $786.16 \pm 5.75^{* * *}$ \\
\hline \multirow{15}{*}{ Longquan no. 1} & B3/72 & $343.08 \pm 7.28$ & $585.50 \pm 24.31 * * *$ & $454.62 \pm 13.52 * * *$ & $623.06 \pm 31.30^{* * *}$ \\
\hline & $\mathrm{B} 3 / 55$ & $352.67 \pm 13.74$ & $660.05 \pm 7.20^{* * *}$ & $524.22 \pm 25.55^{* * *}$ & $737.59 \pm 0.47 * * *$ \\
\hline & $\mathrm{B} 3 / 40$ & $402.38 \pm 25.24 * * *$ & $553.67 \pm 7.28 * * *$ & $437.09 \pm 8.32 * * *$ & $665.26 \pm 10.34 * * *$ \\
\hline & B3/65 & $354.42 \pm 13.74$ & $563.26 \pm 9.46^{* * *}$ & $478.46 \pm 12.48^{* * *}$ & $497.30 \pm 6.99$ \\
\hline & $\mathrm{B} 3 / 70$ & $383.63 \pm 17.00 * * *$ & $777.77 \pm 43.33 * * *$ & $459.01 \pm 12.76^{* * *}$ & $839.52 \pm 11.92 * * *$ \\
\hline & $\mathrm{B} 3 / 37$ & $370.11 \pm 17.02 * *$ & $657.00 \pm 2.62 * * *$ & $409.14 \pm 3.11 * * *$ & $648.82 \pm 8.71^{* * *}$ \\
\hline & $\mathrm{B} 3 / 45$ & $405.86 \pm 16.39^{* * *}$ & $636.94 \pm 13.99 * * *$ & $433.52 \pm 1.64^{* * *}$ & $628.82 \pm 0.47^{* * *}$ \\
\hline & $\mathrm{B} 4 / 14$ & $413.28 \pm 16.14 * * *$ & $609.91 \pm 7.28^{* * *}$ & $379.00 \pm 23.77^{* *}$ & $709.37 \pm 15.97^{* * *}$ \\
\hline & B4/3/31 & $486.09 \pm 4.95 * * *$ & $551.92 \pm 16.67 * * *$ & $425.30 \pm 3.58^{* * *}$ & $612.65 \pm 16.16^{* * *}$ \\
\hline & B3/56 & $401.94 \pm 12.48^{* * *}$ & $728.07 \pm 28.82 * * *$ & $482.30 \pm 6.59^{* * *}$ & $755.68 \pm 11.92^{* * *}$ \\
\hline & B3/47 & $373.60 \pm 13.99^{* *}$ & $635.64 \pm 21.02 * * *$ & $417.63 \pm 4.14^{* * *}$ & $680.60 \pm 7.64 * * *$ \\
\hline & B3/39 & $375.34 \pm 16.87 * * *$ & $624.74 \pm 17.02 * * *$ & $508.33 \pm 4.27^{* * *}$ & $786.91 \pm 8.44 * * *$ \\
\hline & $\mathrm{B} 3 / 73$ & $443.36 \pm 15.98^{* * *}$ & $597.70 \pm 9.28 * * *$ & $435.72 \pm 2.51 * * *$ & $631.28 \pm 12.37^{* * *}$ \\
\hline & $\mathrm{B} 3 / 57$ & $441.18 \pm 6.80^{* * *}$ & $681.85 \pm 23.54 * * *$ & $526.14 \pm 11.52 * * *$ & $834.59 \pm 24.86^{* * *}$ \\
\hline & $\mathrm{B} 3 / 53$ & $418.07 \pm 9.82 * * *$ & $593.78 \pm 27.20 * * *$ & $577.37 \pm 14.82 * * *$ & $604.98 \pm 2.51^{* * *}$ \\
\hline \multirow{6}{*}{ Changbai no. 1} & Diploid & $340.03 \pm 13.99$ & $648.28 \pm 8.51$ & $405.30 \pm 4.14$ & $609.98 \pm 5.02$ \\
\hline & Q11 & $326.07 \pm 23.80$ & $527.51 \pm 11.99 * * *$ & $392.15 \pm 1.71$ & $526.96 \pm 12.45^{* * *}$ \\
\hline & Q21 & $429.84 \pm 10.16^{* * *}$ & $588.55 \pm 18.70^{* * *}$ & $452.98 \pm 37.57$ & $614.36 \pm 11.51$ \\
\hline & Q16 & $426.79 \pm 18.17 * * *$ & $508.76 \pm 14.41^{* * *}$ & $528.05 \pm 15.85^{* * *}$ & $546.14 \pm 15.07^{* * *}$ \\
\hline & Q27 & $449.46 \pm 9.82 * * *$ & $566.75 \pm 12.61 * * *$ & $534.08 \pm 35.22 * * *$ & $544.77 \pm 9.91 * * *$ \\
\hline & Q24 & $510.94 \pm 35.52 * * *$ & $656.56 \pm 8.90$ & $625.32 \pm 13.19 * * *$ & $705.61 \pm 7.12^{* * *}$ \\
\hline \multirow{2}{*}{ Ruantiaobaisha } & Diploid & $300.35 \pm 18.97$ & $677.93 \pm 12.61$ & $462.02 \pm 20.57$ & $651.35 \pm 11.12$ \\
\hline & $\mathrm{H} 3 / 24$ & $521.84 \pm 20.11 * * *$ & $571.98 \pm 31.47 * * *$ & $731.36 \pm 19.05^{* * *}$ & $607.79 \pm 13.53^{* *}$ \\
\hline
\end{tabular}

The values represent the mean $\pm \mathrm{SD}(\mathrm{n}=3)$.

Based on Tukey's honestly significant difference test after one-way analysis of variance, statistically significant events compared with corresponding diploids are indicated as $* * *, P<0.01$ and ${ }^{* *}, P<0.05$.

response to polyploidization in triploid tea plants (Das et al., 2013). In this study of loquat, we revealed a significant alteration in the accumulation of total flavonoids and phenolics in the leaves of most triploid genotypes compared with their diploid parents. Contrary to what was anticipated a general increase in the contents of total flavonoids and total phenolics was not observed. Nevertheless, there was a clear tendency toward a significant increase in these secondary metabolites in the triploids when 
Table 4. Correlation coefficients of functional compounds and antioxidant activities of extracts of leaves and flowers buds of loquat plants.

\begin{tabular}{|c|c|c|c|c|}
\hline & \multicolumn{2}{|c|}{ Diploid } & \multicolumn{2}{|c|}{ Triploid } \\
\hline & DPPH & FRAP & DPPH & FRAP \\
\hline Total flavonoids in leaves & $0.60^{* *}$ & $0.85^{* * *}$ & $0.73^{* * *}$ & $0.81 * * *$ \\
\hline Total phenolics in leaves & $0.60 * *$ & $0.91 * * *$ & $0.71 * * *$ & $0.86^{* * *}$ \\
\hline Total flavonoids in flower buds & $0.86^{* * *}$ & $0.83 * * *$ & $0.66 * *$ & $0.63 * *$ \\
\hline Total phenolics in flower buds & $0.71 * * *$ & $0.53 * *$ & $0.65 * *$ & $0.64 * *$ \\
\hline
\end{tabular}

**Significant correlation at $P<0.05$. ***Significant correlation at $P<0.01$.

they were poorly accumulated in diploids. For those diploids with relatively high accumulations of total flavonoids and phenolics, further accumulations with chromosome doubling are difficult.

Our research demonstrated that the accumulation of metabolites in triploid loquat is affected by not only the increase in gene dosage through the change in chromosome numbers but also the genetic background of the donor genotype. Nevertheless, through this work, we have been able to select triploid loquat plants with high accumulations of flavonoids and phenolics in leaves and flower buds. The contents of the total flavonoids and phenolics in the leaves of the triploid loquat ' $\mathrm{H} 3 / 24$ ' were recorded as $212.00 \mathrm{mg} \mathrm{RE} / \mathrm{g}$ DW and $93.06 \mathrm{mg} \mathrm{GAE} / \mathrm{g}$ DW, respectively. These values are significantly higher than those of wild loquat (Hong et al., 2008a). Our results were in contrast to those of a previous study that reported that wild loquats generally have higher contents of flavonoids and phenolics in leaves relative to cultivars (Hong et al., 2008a). Triploidization has not only led to the enlargement of leaves and flowers (Liang et al., 2011a, b) but also resulted in the enhancement of flavonoids and phenolics accumulations on DW basis. Such findings are particularly encouraging and have provided the foundation for genetic improvements in the contents of the flavonoids and phenolics through selection breeding.

The mechanism of the often-reported enhancement in secondary metabolism in plants through polyploidization is unclear. Polyploidization is usually associated with an increased organ and cell sizes (Dudits et al., 2016; Levan, 1939; Yu et al., 2010). The increased cell size of polyploids affects enzyme activity by offering a positive space advantage to polyploids for cell surfacerelated activities (Galitski et al., 1999; Lavania et al., 2012), which may be attributable to the upregulated expression of some key enzyme genes relevant to the secondary metabolite biosynthetic pathway (Lin et al., 2011). Because the leaves of the triploid loquat have bigger mesophyll cells than those of the diploid loquat (data not shown), it will be particularly interesting to investigate other key enzyme activities in triploid loquat relevant to the biosynthesis of flavonoids and phenolics in the future.

The increase in gene expression and gene dosage as a result of polyploidization might be attributable to the observed variations in the contents of flavonoids and phenolics and antioxidant activities. For instance, the in- creased cytosine methylation was found to be related to the enhanced accumulation of secondary metabolites in the autopolyploid aromatic Cymbopogon grasses (Lavania et al., 2012). Upregulated gene expression in the metabolite pathways of a number of bioactive compounds was believed to be responsible for the increased biomass and targeted metabolites in induced autotetraploid Chinese woad (Isatis indigotica Fort.) (Zhou et al., 2015). Therefore, it is necessary to investigate and integrate the loquat genome, transcriptome, and metabolome in future work to elucidate the mechanisms of triploidization-enhanced secondary metabolism in triploid loquat.

In loquat, the antioxidation activity is mainly determined by the content of its antioxidant components, such as flavonoids and phenolics in leaves, flowers, and fruits (Esmaeili et al., 2014; Ferreres et al., 2009; Hong et al., 2008a; Xu and Chen, 2011; Zhou et al., 2011). In this work, we confirmed that the antioxidant activity of leaves and flower buds in diploid loquat and the corresponding triploid loquat were significantly and positively correlated with the contents of flavonoids and phenolics. Moreover, we found a stronger positive correlation between antioxidant activity and the contents of flavonoids and phenolics in leaf extracts of triploid loquat compared with the diploid parent (Table 4). Therefore, the variations in antioxidant activity observed in the leaves of triploid loquat were largely attributable to the variations in the contents of flavonoids and phenolics. This was in contrast to the flower extracts of triploid loquat, which showed a relatively weaker correlation between antioxidant activity and the total contents of flavonoids and phenolics when compared with diploids. It is likely that antioxidant compounds other than flavonoids and phenolics may have changed during triploidization. In diploid loquat leaves, several flavonoids and phenolics compounds were previously identified, including chlorogenic acid, quercetin-3-sambubioside, methyl chlorogenate, kaempferol-3-rhamnoside, quercetin-3rhamnoside, cinchonain Ib, cinchonain Ia, epicatechin, quercetin-3-O- $\alpha$-L-rhamnoside, kaempferol, galangin, hesperidin, hyperoside, kaempferol-3, 7-diglucoside, isoquercetin, and rutin (Hong et al., 2008b; Jung et al., 1999; Liu et al., 2014). In loquat flower extract, the major antioxidants were hesperetin and gallic acid (Esmaeili et al., 2014). Further studies including a detailed analysis of antioxidant composition and the metabolic pathway and a transcriptomic analysis of triploid loquat may help to elucidate the biochemical mechanisms of antioxidant activity and how it was affected by the ploidy level in loquat.

\section{Conclusion}

In this study, we demonstrated that the contents of total flavonoids and phenolics in leaves and flower buds of triploid loquat were variable compared with those of their respective diploid parents, and that this could be attributed to not only an increased number of chromosomes but also parental genetic diversity. Furthermore, we identified some triploid loquat genotypes that showed significantly higher accumulations of total flavonoids and phenolics and stronger antioxidant activities in leaves and flower buds relative to their respective diploid parents. In triploid loquat leaves, a strong correlation between antioxidant activities and contents of total flavonoids and phenolics has been established. The higher contents of total flavonoids and phenolics and stronger antioxidant activity identified in this study may be combined with other already established triploid traits, such as larger vegetative organs and better tolerance to various stresses in triploid loquat, as a feasible strategy for breeding loquat cultivars with high pharmaceutical potency.

\section{Literature Cited}

Benzie, I.F. and J.J. Strain. 1996. The ferric reducing ability of plasma (FRAP) as a measure of antioxidant power: The FRAP assay. Anal. Biochem. 239:70-76, doi: 10.1006/abio.1996. 0292 .

Brand-Williams, W., M. Cuvelier, and C. Berset. 1995. Use of a free radical method to evaluate antioxidant activity. Lebensm. Wiss. Technol. 28:25-30.

Bretagnolle, F. and J.D. Thompson. 1995. Gametes with the somatic chromosome number: Mechanisms of their formation and role in the evolution of autopolyploid plants. New Phytol. 129:1-22.

Caruso, I., F. Dal Piaz, N. Malafronte, N. De Tommasi, R. Aversano, C.W. Zottele, M.T Scarano, and D. Carputo. 2013. Impact of ploidy change on secondary metabolites and photochemical efficiency in Solanum bulbocastanum. Nat. Prod. Commun. 8:1387-1392.

Chinese Pharmacopeia Commission. 2015. Pharmacopoeia of the People's Republic of China, p. 204-205. Chemistry Industry Publishing House, Beijing, China.

Cohen, H., A. Fait, and N. Tel-Zur. 2013. Morphological, cytological and metabolic consequences of autopolyploidization in Hylocereus (Cactaceae) species. BMC Plant Biol. 13:173.

Das, S.K., S. Sabhapondi, G. Ahmed, and S. Das. 2013. Biochemical evaluation of triploid progenies of diploid $\times$ tetraploid breeding populations of Camellia for genotypes rich in catechin and caffeine. Biochem. Genet. 51:358-376.

Dudits, D., K. Török, A. Cseri, K. Paul, A.V. Nagy, B. Nagy, L. Sass, G. Ferenc, R. Vankova, P. Dobrev, I. Vass, and F. Ayaydin. 2016. Response of organ structure and physiology to autotetraploidization in early development of energy willow Salix viminalis. Plant Physiol. 170:1504-1523. 
Esmaeili, A.H., A. Hajizadeh Moghaddam, and M.J. Chaichi. 2014. Identification, determination, and study of antioxidative activities of hesperetin and gallic acid in hydro-alcoholic extract from flowers of Eriobotrya japonica (Lindl.). Avicenna J. Phytomed. 4:260-266.

Ferreres, F., D. Gomes, P. Valentão, R. Gonçalves, R. Pio, E.A. Chagas, R.M. Seabra, and P.B. Andrade. 2009. Improved loquat (Eriobotrya japonica Lindl.) cultivars: Variation of phenolics and antioxidative potential. Food Chem. 114:1019-1027.

Galitski, T., A.J. Saldanha, C.A. Styles, E.S. Lander, and G.R. Fink. 1999. Ploidy regulation of gene expression. Science 285:251-254.

Guo, Q. G., X. L. Li, W. X. Wang, Q. He, and G.L. Liang. 2007. Occurrence of natural triploids in loquat. Acta Hort. 750:125-128.

Hong, Y., S. Lin, Y. Jiang, and M. Ashraf. 2008a. Variation in contents of total phenolics and flavonoids and antioxidant activities in the leaves of 11 Eriobotrya species. Plant Foods Hum. Nutr. 63:200-204.

Hong, Y., Y. Qiao, S. Lin, Y. Jiang, and F. Chen. 2008b. Characterization of antioxidant compounds in Eriobotrya fragrans Champ leaf. Scientia Hort. 118:288-292.

Huang, Y., J. Li, Q. Cao, S.C. Yu, X.W. Lv, Y. Jin, L. Zhang, Y.H. Zou, and J.F. Ge. 2006. Antioxidative effect of triterpene acids of Eriobotrya japonica (Thunb.) Lindl. leaf in chronic bronchitis rats. Life Sci. 78:2749-2757.

Jia, Z., M. Tang, and J. Wu. 1999. The determination of flavonoid contents in mulberry and their Scavenging effects on superoxide radicals. Food Chem. 64:555-559.

Jung, H.A., J.C. Park, H.Y. Chung, J. Kim, and J.S. Choi. 1999. Antioxidant flavonoids and chlorogenic acid from the leaves of Eriobotrya japonica. Arch. Pharm. Res. 22:213-218.

Kaensaksiri, T., P. Soontornchainaksaeng, N. Soonthornchareonnon, and S. Prathanturarug. 2011. In vitro induction of polyploidy in Centella asiatica (L.) Urban. Plant Cell Tissue Organ Cult. 107:187-194.

Kikuchi, S., M. Iwasuna, A. Kobori, Y. Tsutaki, A. Yoshida, Y. Murota, E. Nishino, H. Sassa, and T. Koba. 2014. Seed formation in triploid loquat (Eriobotrya japonica) through crosshybridization with pollen of diploid cultivars. Breed. Sci. 64:176-182.

Kong, D., Y. Li, M. Bai, Y. Deng, G. Liang, and H. Wu. 2017. A comparative study of the dynamic accumulation of polyphenol components and the changes in their antioxidant activities in diploid and tetraploid Lonicera japonica. Plant Physiol. Biochem. 112:87-96.

Kovalsky, I.E., J.M. Roggero Luque, G. Elías, S.A. Fernández, and V.G. Solís Neffa. 2017.
The role of triploids in the origin and evolution of polyploids of Turnera sidoides complex (Passifloraceae, Turneroideae). J. Plant Res. 10:1-13.

Lavania, U.C., S. Srivastava, S. Lavania, S. Basu, N.K. Misra, and Y. Mukai. 2012. Autopolyploidy differentially influences body size in plants, but facilitates enhanced accumulation of secondary metabolites, causing increased cytosine methylation. Plant J. 71:539-549.

Levan, A. 1939. Tetraploidy and octoploidy induced by colchicine in diploid Petunia. Hereditas 25:109-131.

Liang, G.L. 2006. Selection natural triploid loquats and studies on their genetic characters and genome analysis. Southwest University, Chongqing, $\mathrm{PhD}$ Diss.

Liang, G.L., W.X. Wang, X.L. Li, Q.G. Guo, S.Q. Xiang, and Q. He. 2011a. Selection of largefruited triploid plant of loquat. Acta Hort. 887:95-100.

Liang, G.L., W.X. Wang, S.Q. Xiang, Q.G. Guo, X.L. Li, and Q. He. 2011b. Morphological comparing between diploid and triploid loquat. Acta Hort. 887:261-264.

Liang, S.L., J.B. Dang, G.L. Liang, and Q.G. Guo. 2018. Meiosis observation and fertility analysis in natural tetraploid loquat of 'B431'. Acta Hort. Sinica 45:1895-1904. (In Chinese).

Lin, S., X. Huang, J. Cuevas, and J. Janick. 2007. Loquat: An ancient fruit crop with a promising future. Chronica Horticulture 47:12-15.

Lin, X., Y. Zhou, J. Zhang, X. Lu, F. Zhang, Q. Shen, S. Wu, Y. Chen, T. Wang, and K. Tang. 2011. Enhancement of artemisinin content in tetraploid Artemisia annua plants by modulating the expression of genes in artemisinin biosynthetic pathway. Biotechnol. Appl. Biochem. 58:50-70.

Liu, H., X. Yu, J. Cheng, and W. Li. 2014. Studies on the flavonoids from leaf of Eriobotrya japonica (Thunb.) Lindl. Chinese Traditional Patent Medicine 36:330-332. (In Chinese).

Liu, Y., W. Zhang, C. Xu, and X. Li. 2016. Biological activities of extracts from loquat (Eriobotrya japonica Lindl.): A review. Intl. J. Mol. Sci. 17:1983.

Nakasone, Y., M. Yonaha, K. Wada, S. Adaniya, H. Kikuzaki, and N. Nakatani. 1999. Evaluation of pungency in the diploid and tetraploid types of ginger (Zingiber officinale Roscoe) and antioxidative activity of their methanol extracts. Jpn. J. Trop. Agr. 43:71-75.

Ramsey, J. and D.W. Schemske. 1998. Pathways, mechanisms, and rates of polyploid formation in flowering plants. Annu. Rev. Ecol. Syst. 29:467-501.

Singleton, V.L., R. Orthofer, and R.M. LamuelaRaventos. 1999. Analysis of total phenols and other oxidation substrates and antioxidants by means of Folin-Ciocalteu reagent. Methods Enzymol. 299:152-178.

Sun, Q., H. Sun, R.L. Bell, H. Li, and L. Xin. 2011. Variation of phenotype, ploidy level, and organogenic potential of in vitro regenerated polyploids of Pyrus communis. Plant Cell Tissue Organ Cult. 107:131-140.

Toda, E. and T. Okamoto. 2016. Formation of triploid plants via possible polyspermy. Plant Signal. Behav. 11:e1218107.

Van Laere, K., S.C. Franca, H. Vansteenkiste, J. Van Huylenbroeck, K. Steppe, and M.C. Van Labeke. 2011. Influence of ploidy level on morphology, growth and drought susceptibility in Spathiphyllum wallisii. Acta Physiol. Plant. 33:1149-1156.

Wang, W.X., Q.G. Guo, S.Q. Xiang, L.X. Li, and G.L. Liang. 2003. Study on the effect of hotshock treatment on the occurrence frequency of 2 pollen of loquat tree. Fruit of Sci. 20:284286. (In Chinese).

Wood, T.E., N. Takebayashi, M.S. Barker, I. Mayrose, P.B. Greenspoon, and L.H. Rieseberg. 2009. The frequency of polyploid speciation in vascular plants. Proc. Natl. Acad. Sci. USA 106:13875-13879.

Xie, Z., H. Huang, Y. Zhao, H. Shi, S. Wang, T.T. Wang, P. Chen, and L.L. Yu. 2012. Chemical composition and anti-proliferative and antiinflammatory effects of the leaf and whole-plant samples of diploid and tetraploid Gynostemma pentaphyllum (Thunb.) Makino. Food Chem. 132:125-133.

Xu, H. and J. Chen. 2011. Commercial quality, major bioactive compound content and antioxidant capacity of 12 cultivars of loquat (Eriobotrya japonica Lindl.) fruits. J. Sci. Food Agr. 91:1057-1063.

Yu, Z., G. Haberer, M. Matthes, T. Rattei, K.F. Mayer, A. Gierl, and R.A. Torres-Ruiz. 2010. Impact of natural genetic variation on the transcriptome of autotetraploid Arabidopsis thaliana. Proc. Natl. Acad. Sci. USA 107:17809-17814.

Zahedi, A.A., B. Hosseini, M. Fattahi, E. Dehghan, H. Parastar, and H. Madani. 2014. Overproduction valuable methoxylated flavones in induced tetraploid plants of Dracocephalum kotschy Boiss. Bot. Stud. 55:22.

Zhou, C., C. Sun, K. Chen, and X. Li. 2011. Flavonoids, phenolics, and antioxidant capacity in the flower of Eriobotrya japonica Lindl. Intl. J. Mol. Sci. 12:2935-2945.

Zhou, Y., L. Kang, S. Liao, Q. Pan, X. Ge, and Z. Li. 2015. Transcriptomic analysis reveals differential gene expressions for cell growth and functional secondary metabolites in induced autotetraploid of Chinese woad (Isatis indigotica Fort.). PLoS One 10(3):e0116392. 

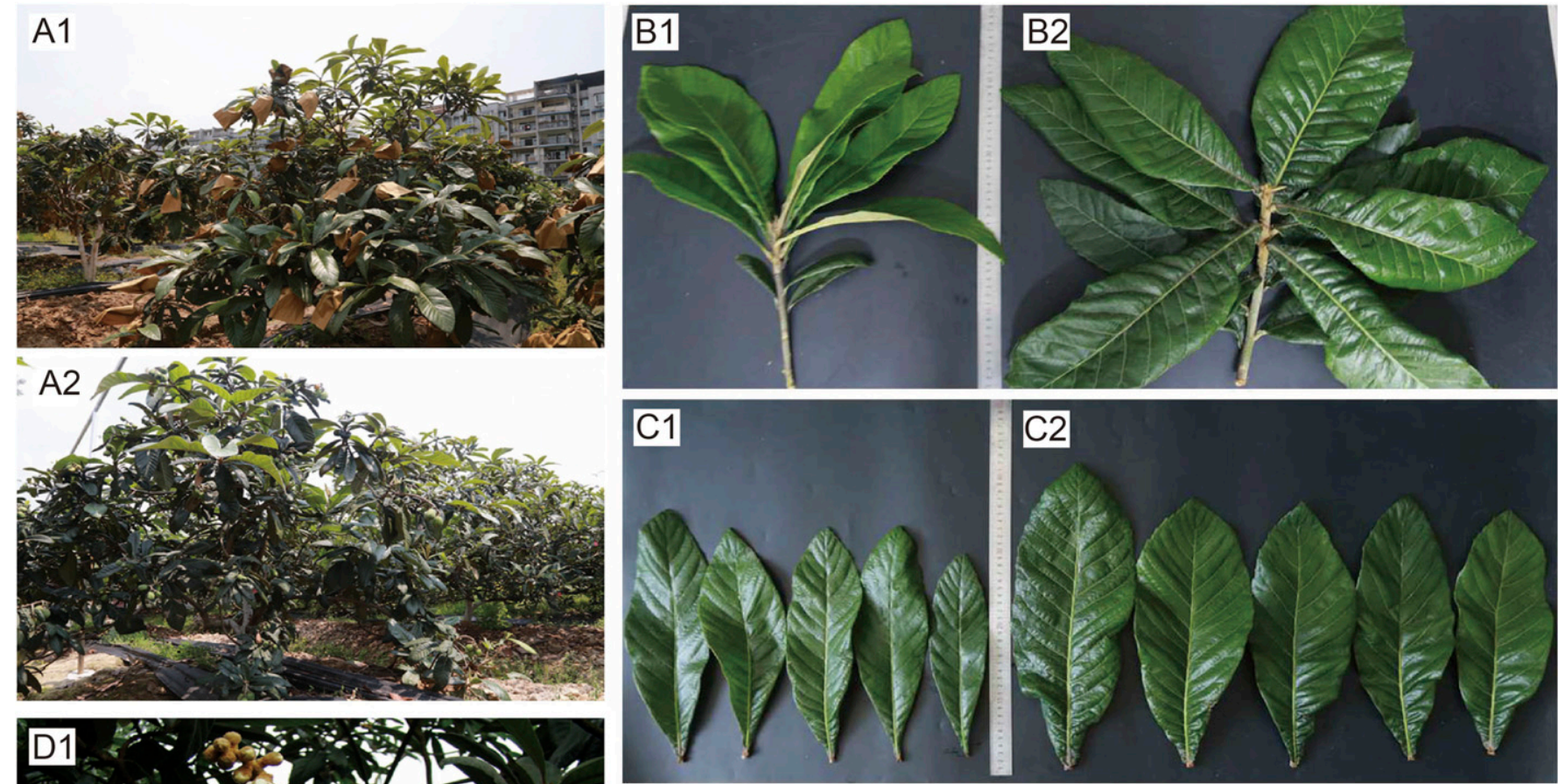

$\mathrm{C} 2$
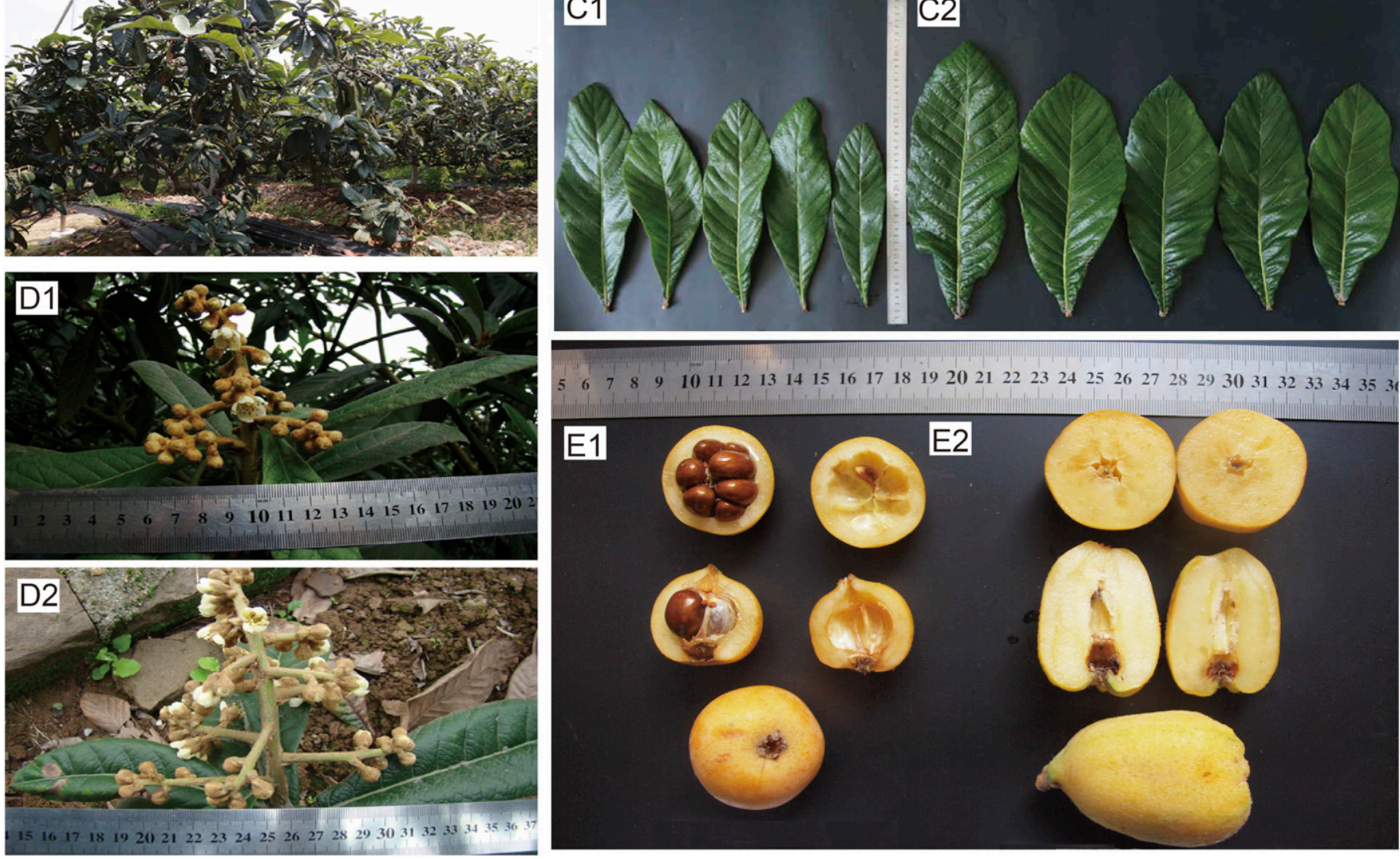

Supplemental Fig. 1. The trees, shoots, leaves, flowers, and fruits of the triploid loquat genotype 'Q27' and corresponding diploid loquat 'Changbai no. 1'. (A1) Tree of 'Changbai no. 1'. (A2) Tree of 'Q27'. (B1) Shoots of 'Changbai no. 1'. (B2) Shoots of 'Q27'. (C1) Leaves of 'Changbai no. 1'. (C2) Leaves of 'Q27'. (D1) Flowers of 'Changbai no. 1'. (D2) Flowers of 'Q27'. (E1) Fruits of 'Changbai no. 1'. (E2) Fruits of 'Q27'. 

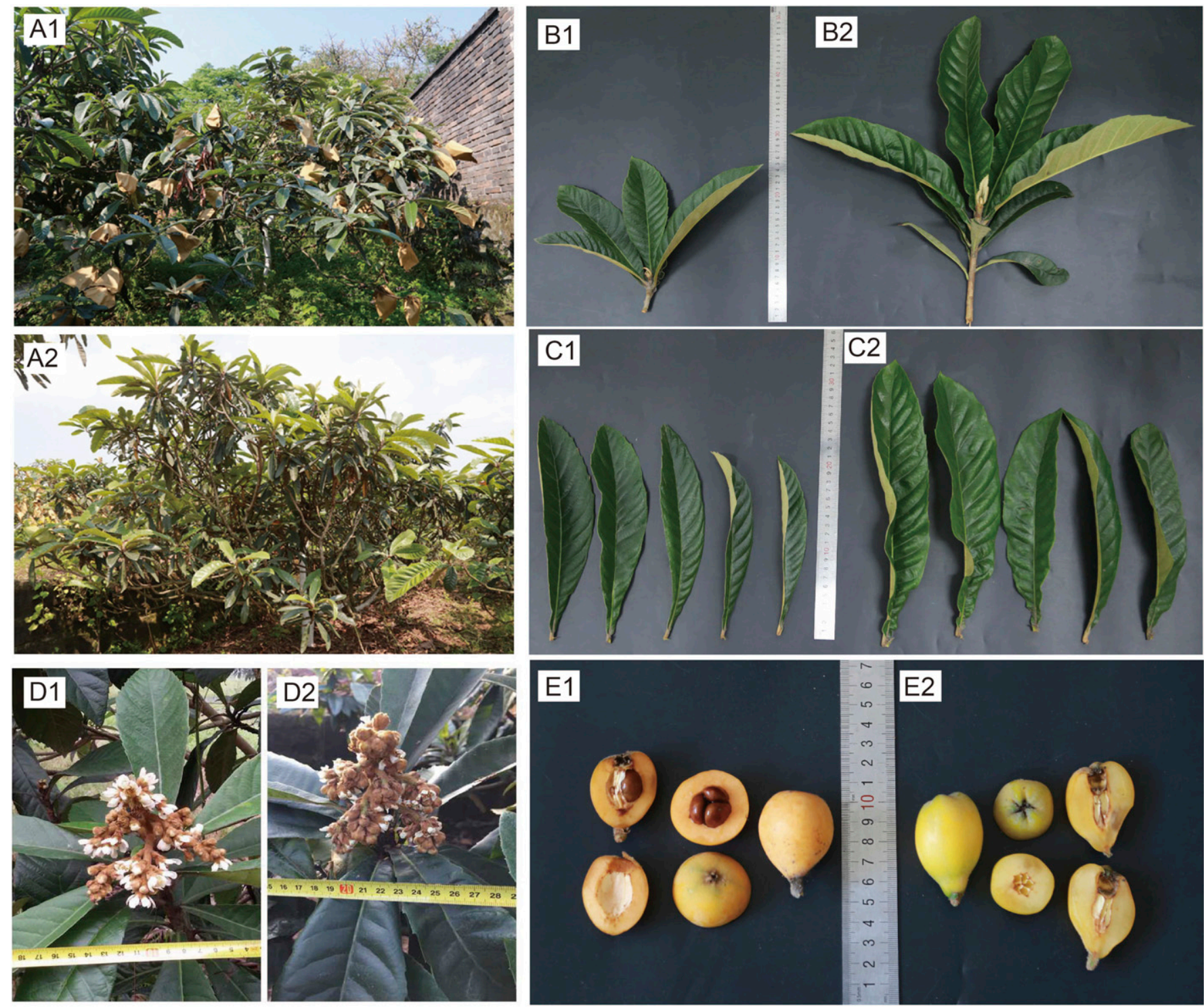

Supplemental Fig. 2. Trees, shoots, leaves, flowers, and fruits of the triploid loquat genotype 'K3/59' and corresponding diploid loquat '77-1'. (A1) Tree of ' $77-1$ '. (A2) Tree of 'K3/59'. (B1) Shoots of '77-1'. (B2) Shoots of 'K3/59'. (C1) Leaves of '77-1'. (C2) Leaves of 'K3/59'. (D1) Flowers of '77-1'. (D2) Flowers of 'K3/59'. (E1) Fruits of ' $77-1$ '. (E2) Fruits of 'K3/59'. 

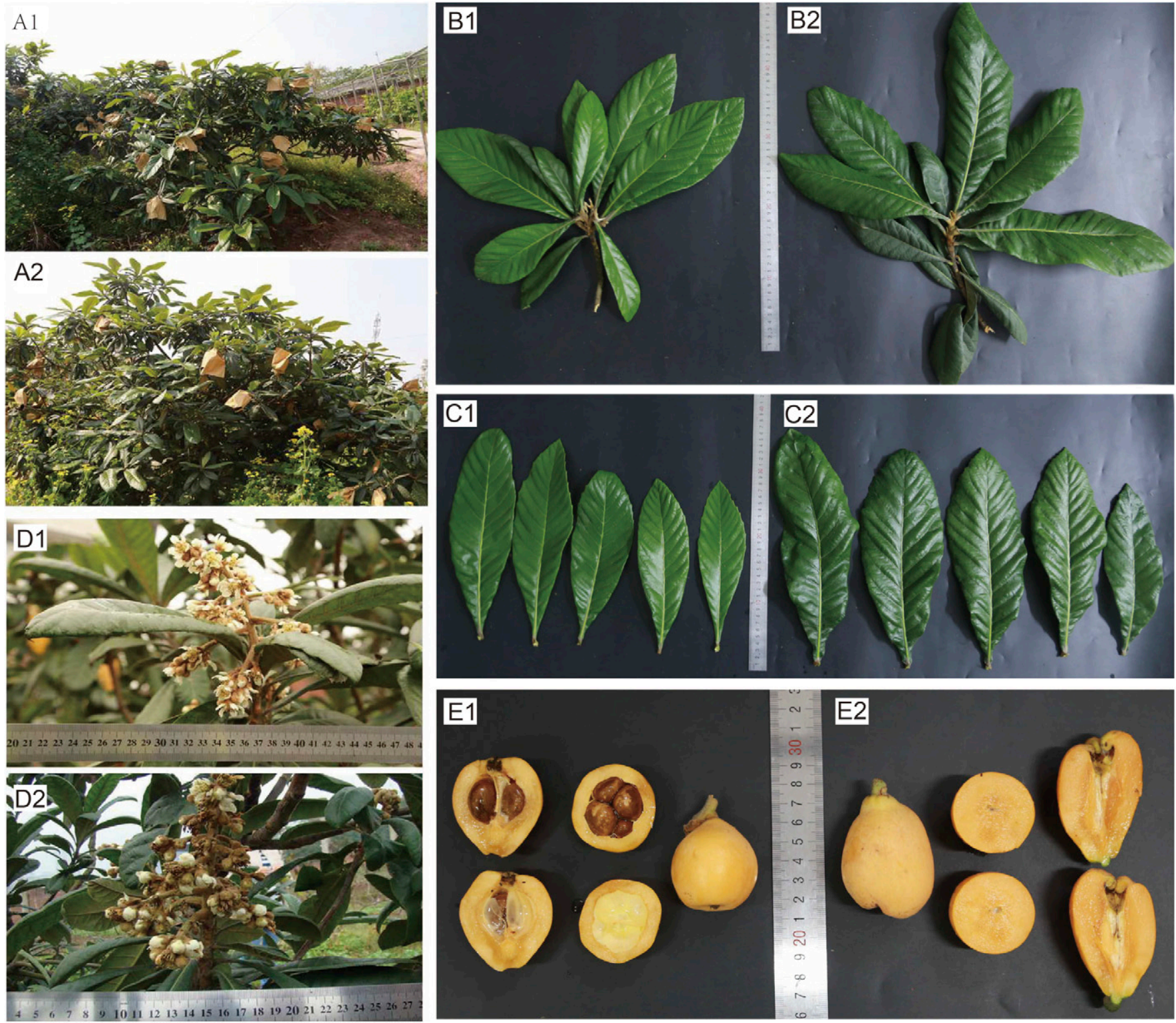

Supplemental Fig. 3. Trees, shoots, leaves, flowers, and fruits of the triploid loquat genotype 'Jinfeng' and corresponding diploid loquat 'D4/25'. (A1) Tree of 'Jinfeng'. (A2) Tree of 'D4/25'. (B1) Shoots of 'Jinfeng'. (B2) Shoots of 'D4/25'. (C1) Leaves of 'Jinfeng'. (C2) Leaves of 'D4/25'. (D1) Flowers of 'Jinfeng'. (D2) Flowers of 'D4/25'. (E1) Fruits of 'Jinfeng'. (E2) Fruits of 'D4/25'. 

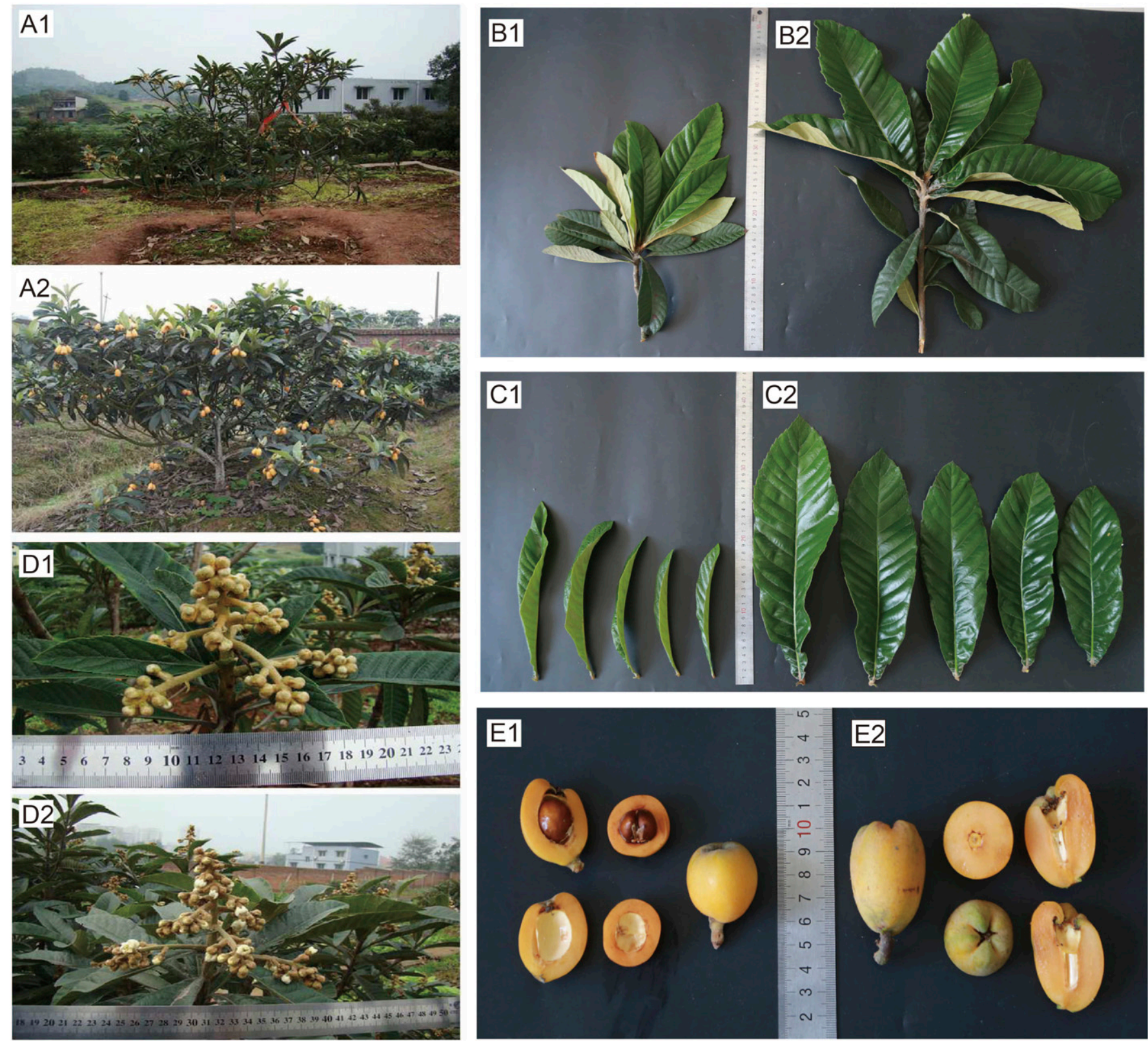

Supplemental Fig. 4. Trees, shoots, leaves, flowers, and fruits of the triploid loquat genotype 'Dawuxing' and corresponding diploid loquat 'A3/22'. (A1) Tree of 'Dawuxing'. (A2) Tree of 'A3/22'. (B1) Shoots of 'Dawuxing'. (B2) Shoots of 'A3/22'. (C1) Leaves of 'Dawuxing'. (C2) Leaves of 'A3/22'. (D1) Flowers of 'Dawuxing'. (D2) Flowers of 'A3/22'. (E1) Fruits of 'Dawuxing'. (E2) Fruits of 'A3/22'. 


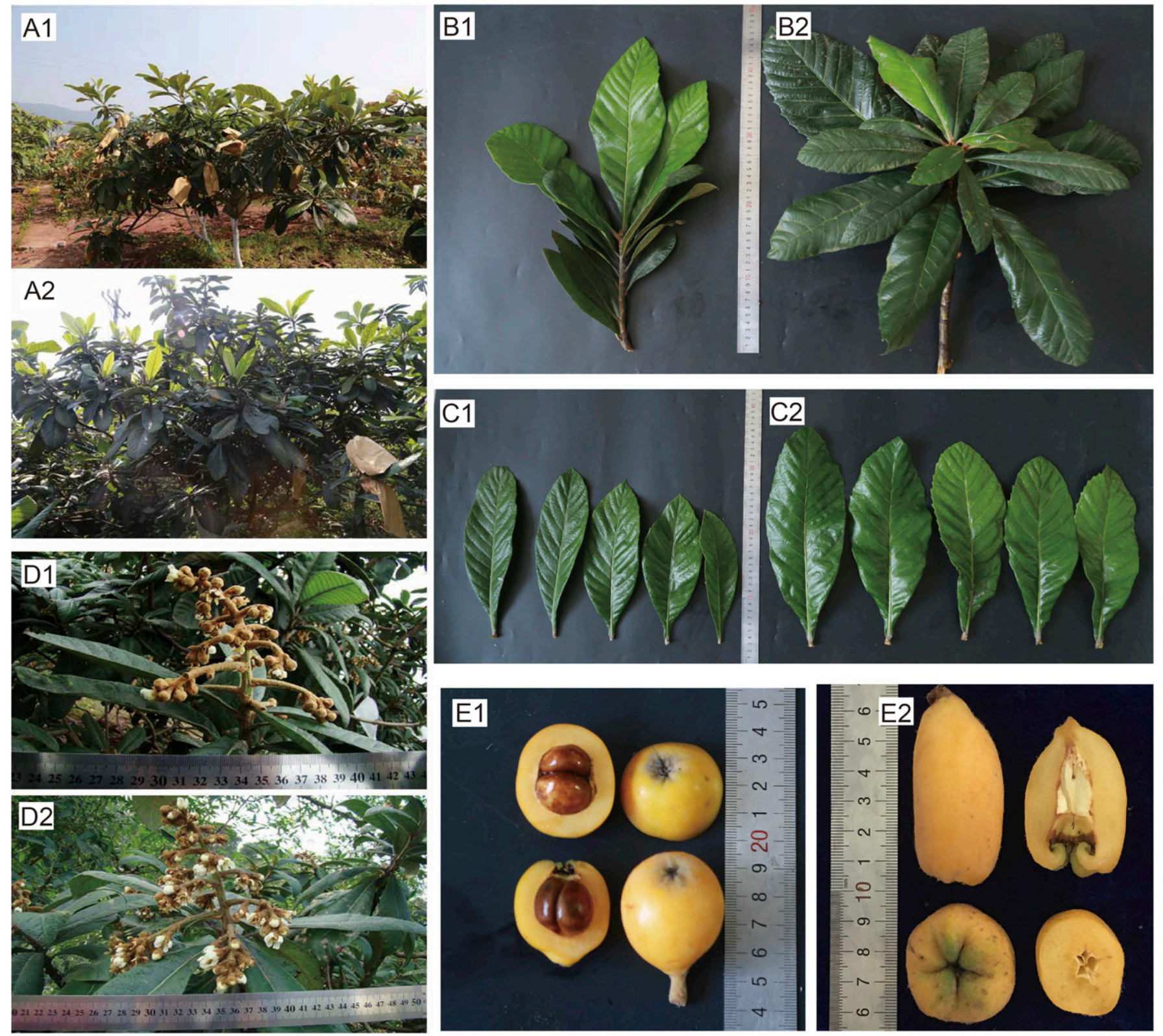

Supplemental Fig. 5. Trees, shoots, leaves, flowers, and fruits of the triploid loquat genotype 'Xiangzhong' and corresponding diploid loquat 'N17'. (A1) Tree of

'Xiangzhong'. (A2) Tree of 'N17'. (B1) Shoots of 'Xiangzhong'. (B2) Shoots of 'N17'. (C1) Leaves of 'Xiangzhong'. (C2) Leaves of 'N17'. (D1) Flowers of 'Xiangzhong'. (D2) Flowers of 'N17'. (E1) Fruits of 'Xiangzhong'. (E2) Fruits of 'N17'. 

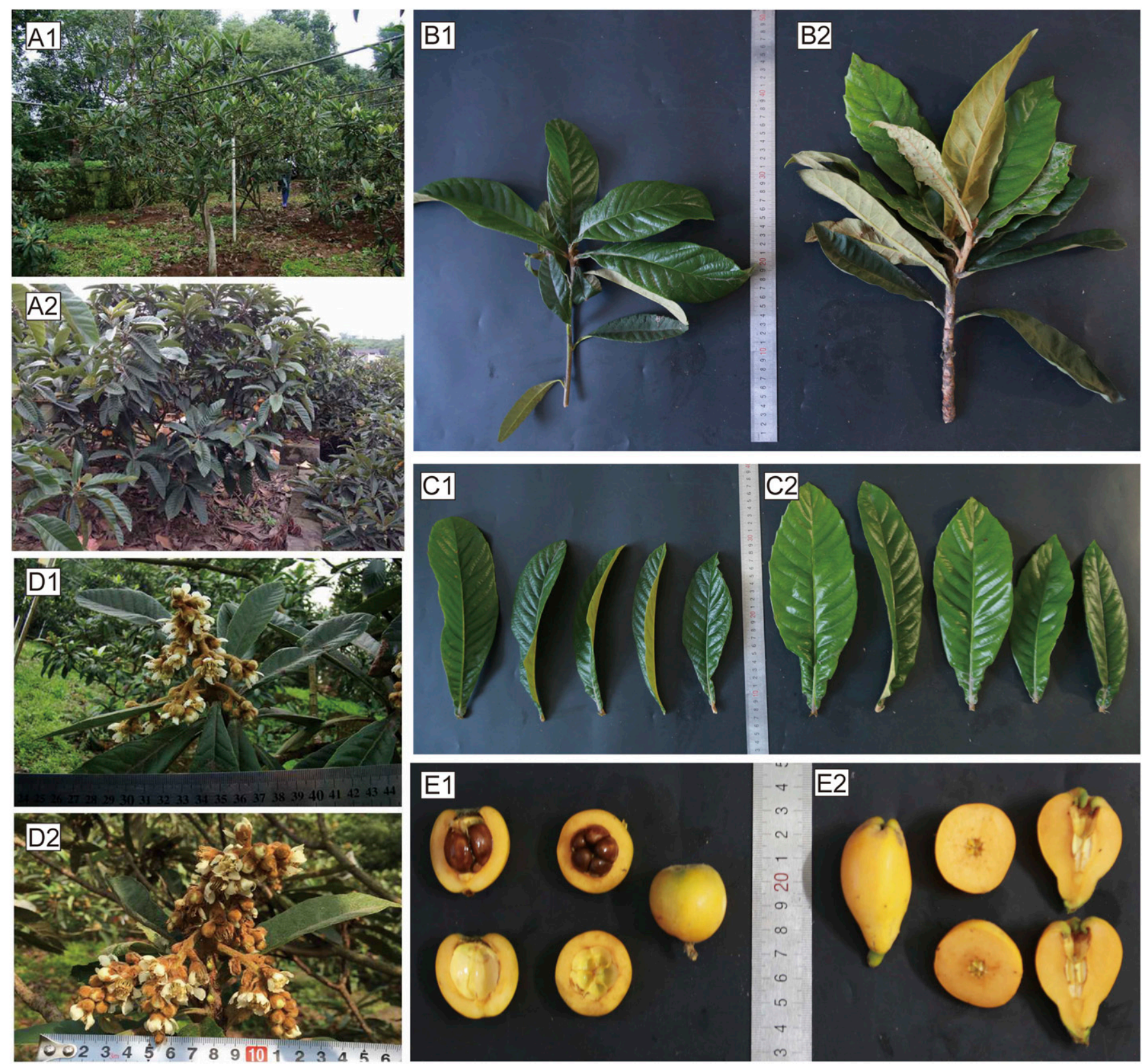

Supplemental Fig. 6. Trees, shoots, leaves, flowers, and fruits of the triploid loquat genotype 'Longquan no. 1' and corresponding diploid loquat 'B3/53'. (A1) Tree of 'Longquan no. 1'. (A2) Tree of 'B3/53'. (B1) Shoots of 'Longquan no. 1'. (B2) Shoots of 'B3/53'. (C1) Leaves of 'Longquan no. 1'. (C2) Leaves of 'B3/53'. (D1) Flowers of 'Longquan no. 1'. (D2) Flowers of 'B3/53'. (E1) Fruits of 'Longquan no. 1'. (E2) Fruits of 'B3/53'. 